\title{
Comparação entre a mecânica relativista e a mecânica newtoniana
}

(A comparison between the relativistic and the Newtonian mechanics)

\author{
G.F. Leal Ferreira
}

\author{
Instituto de Física de São Carlos, D.F.C.M., São Carlos, SP, Brasil. \\ Recebido em 7/5/03; Manuscrito revisado recebido em 28/11/03; Aceito em 5/1/04
}

\begin{abstract}
Comparação entre as mecânicas Relativista e Newtoniana é realizada num dado sistema de coordenadas, sem nenhuma preocupação com eventuais outros sistemas de coordenadas inerciais em movimento relativo, o que permite percepção mais direta das diferenças entre elas. Toma-se como ponto de partida a equivalência entre massa e energia e chega-se, como anteriormente realizado por T. Theodorsen, à correta dependência entre massa e velocidade.
\end{abstract}

Palvras-chave: Mecânica Clássica, Mecânica Relativística, equivalência massa-energia.

A comparison between the relativistic and the Newtonian mechanics is carried out inside a single coordinate system without reference to other coordinate systems moving relatively to ours. This allows a more direct understanding of the differences between the two mechanics. As a starting point, the equivalence between mass and energy is assumed and taking the route suggested by $\mathrm{T}$. Theodorsen, the correct dependence of the mass on the velocity is obtained.

Keywords: Classical Mechanics, Relativistic Mechanics, mass-energy equilavence.

\section{Introdução}

A Teoria da Relatividade, como usualmente apresentada, dá especial ênfase às mudanças de sistemas de coordenadas, em razão de suas origens. Mesmo um dos seus principais resultados dinâmicos, o da variação da massa com a velocidade, está, na maioria das apresentações, ligado, direta ou indiretamente [1,2], àquelas trocas de sistema de coordenadas. Isto é inconveniente já que as modificações parecem assim se dever unicamente a mudanças de sistemas de coordenadas, quando, na prática, elas ocorrem aquí mesmo, nos nossos laboratórios. E o presente artigo não tem nada de original a não ser esquecer outros sistemas de coordenadas e dar ênfase às mudanças entre a formulação newtoniana, em que a massa é constante (e o conceito de energia é acessório), e a relativista em que tal não ocorre. Para isto, estaremos nos valendo de formulação apresentada em [3], cujo ponto de partida é o da equivalência entre massa e energia, como defendido por Sandin [4] na controvérsia sobre o significado de $m$ na famosa relação $E=m c^{2}$, se constante ou variável, discutido em [5]. Em palavras mais simples, se a 'inércia' depende ou não da velocidade. Como operamos num único sistema de coordenadas, constatamos que $m$ depende de $v$, sem outras filosofias.

\section{Mecânica newtoniana}

A Mecânica Newtoniana da massa pontual constante, $m$, é dada simplesmente por

$$
\vec{F}=\frac{d \vec{p}}{d t}, \operatorname{com} \vec{p}=m \vec{v}
$$

Enviar correspondência para G.F. Leal Ferreira. E-mail: guilherm@if.sc. usp.br. sendo $\vec{F}$ a força, $\vec{p}$ o momento, $\vec{v}$ a velocidade, e $t$ o tempo. $\mathrm{O}$ conceito de energia é na Mecânica Newtoniana um conceito derivado e é obtido da Eq. 1 multiplicando-a escalarmente por $\vec{v}$

$$
d W=\vec{F} \cdot \vec{v} d t=\vec{F} \cdot d \vec{s}=m \vec{v} \cdot d \vec{v}=\frac{m}{2} d \vec{v}^{2}=d E_{c}
$$

em que $W$ é o trabalho de $\vec{F}, d \vec{s}$ o deslocamento em $d t$ e $E_{c}$ a energia cinética. Alternativamente, o conceito de energia, de alto teor físico, é usado como primitivo nas formulações lagrangiana e hamiltoniana, completado pelo conceito de energia potencial.

\subsection{Mecânica newtoniana da massa variável}

A Mecânica Newtoniana também sabe tratar sistemas em que a massa é variável. Em especial, se a massa é adicionada ao sistema do repouso, a Eq. 1 é ainda válida com $m=m(t)$. Já a Eq. 2 tornar-se-ia

$$
d W=\vec{F} \cdot \vec{v} d t=\vec{F} \cdot d \vec{s}=m(t) \vec{v} \cdot d \vec{v}+\vec{v}^{2} d m(t),
$$

$d W$ não sendo mais uma diferencial exata da energia cinética, como é no caso em que a massa é constante. Temos para $d E_{c}$,

$$
d E_{c}=\frac{d\left(m(t) v^{2}\right)}{2 d t}=m(t) \vec{v} \cdot d \vec{v}+\frac{v^{2} d m}{2}<d W,
$$

isto é, a variação da energia cinética é menor do que o trabalho: há algo de irreversível no sistema de massa variável Newtoniano. Comparando as Eqs. 3 e 4, vê-se que a perda de energia mecânica diferencial é $v^{2} d m(t) / 2$. 


\section{Mecânica relativista}

Os primeiros desvios à lei de Newton, Eq. 1, foram observados nas experiências de Kaufmann [5], em que a massa dos elétrons, acelerados em campos elétricos e magnéticos, mostrava ser variável com a velocidade. Portanto, devemos esperar, em princípio, não mais uma única equação como no caso newtoniano, mas duas equações, de forma a estabelecer como se dá aquela variação da massa com a velocidade. Para isso, seguiremos [3], que parte da equivalência entre massa $m(v)$ e energia $E(v)[5]$

$$
E(v)=m(v) c^{2}
$$

A Eq. 1 será agora [3]

$$
\vec{F}=\frac{d}{d t} \vec{p}, \quad \operatorname{com} \vec{p}=\frac{E(v)}{c^{2}} \vec{v}
$$

sendo $c$ a velocidade da luz. Segundo ainda [3], vamos impor que o trabalho da força aplicada seja igual ao aumento da energia $E$ :

$$
d W=\vec{F} \cdot \vec{v} d t=\vec{F} \cdot d \vec{s}=d E(v)
$$

Notemos agora que se multiplicarmos a Eq. 6 escalarmente por $\vec{v}$ e usarmos a Eq. 5, obteremos

$$
c^{2} d E=\vec{v} \cdot d(E \vec{v})
$$

equação que pode ser integrada multiplicando-se os dois membros por $E$, fornecendo a dependência de $E$ com a velocidade

$$
E(v)=\frac{E_{0}}{\sqrt{1-v^{2} / c^{2}}}
$$

sendo $E_{0}$ a energia (ou equivalentemente a massa) de repouso. Portanto, a admissão da Eq. 5, ou seja o da equivalência entre a massa e energia, leva à relação correta entre massa relativista e velocidade.

Voltando à Eq. 7, suporte da Eq. 9, ela e as Eqs. 6 se constituem nas equações da Mecânica Relativista. Portanto, a mudança significativa operada na Mecânica Newtoniana é a incorporação do trabalho realizado sobre a massa (energia) à própria massa (energia), de acordo com a Eq. 7, tornando o trabalho $d W$ uma diferencial exata.

\section{A Hamiltoniana e a Lagrangiana relativistas}

Mostraremos agora que as equações da formulação relativista da seção anterior levam às expressões corretas da hamiltoniana e lagrangiana relativista.

Se a força deriva de um potencial $U(\vec{x})$,

$$
\vec{F} \cdot d \vec{s}=-d U(\vec{x}),
$$

e a Eq. 7 se escreve

$$
d(E(v)+U(\vec{x}))=0
$$

definindo a energia total $H$,

$$
H=E(v)+U(\vec{x}),
$$

$H$, sendo a energia total, deve ser a hamiltoniana quando expresso em função de $p_{i}$, e $x_{i}$,componentes de $\vec{p}$, e de $\vec{x}$ (lembremos que a Eq. 6 define o momento em função de $E(v) \vec{v})$. Sendo $\mathrm{H}\left(x_{i}, p_{i}\right)$, devemos então ter

$$
\dot{x}_{i}=\frac{\partial H}{\partial p_{i}} \quad \text { e } \quad \dot{p}_{i}=-\frac{\partial H}{\partial x_{i}}
$$

em que o ponto significa derivada em relação ao tempo. A equação em $\dot{p}_{i}$ reproduz a Eq. 6. Para analisar a equação em $\dot{x}_{i}$, vamos primeiro supor que estamos em uma dimensão. Teríamos

$$
\dot{x}=v=\frac{c^{2} p}{E}=\frac{\partial H}{\partial p}=\frac{\partial E}{\partial p}=\frac{d E}{d p}
$$

em que as Eqs. 6, 12 e13 foram usadas. O terceiro e o sexto termos formam a equação diferencial

$$
\frac{c^{2} p}{E}=\frac{d E}{d p}
$$

que integrada dá

$$
E^{2}=c^{2} p^{2}+E_{0}^{2},
$$

sendo $E_{0}$ a energia de repouso. A Eq. 16 é uma conhecida relação em dinâmica relativista, confirmando que $H$, considerado dependente de $x$ e $p$, é de fato a hamiltoniana.

No caso geral, tridimensional, voltando à Eq. 14, teríamos

$$
\dot{x}_{i}=v_{i} \frac{c^{2} p_{i}}{E}=\frac{\partial H}{\partial p_{i}}=\frac{d E}{d p} \frac{\partial p}{\partial p_{i}},
$$

em que se usa o fato de $E$ ser função de $p$, módulo de $\vec{p}$. Elevando ao quadrado e somando nas componentes, chega-se à Eq. 15 e daí à Eq. 16, levando em conta que os $\partial p / \partial p_{i}$ são coeficientes angulares.

A lagrangiana $L\left(x_{i}, v_{i}\right)$ é obtida pelo procedimento usual, [2].

$$
L=\sum p_{i} \dot{x}_{i}-H=-\left(E_{0} \sqrt{1-\frac{v^{2}}{c^{2}}}+U(\vec{x})\right)
$$

em que empregamos as Eqs. 6, 9 e 12.

\section{Uma comparação}

Somos tentados a comparar a Mecânica Relativista com a newtoniana de massa varíável, tratada na seção 2.1. Como a diferença entre elas está em que o trabalho nesta última não é reversível, Eq. 4, poderíamos especular, na tentativa de dar ao tratamento relativista a mesma interpretação que no newtoniano, que o trabalho perdido (ver abaixo da Eq. 4) $v^{2} d m(v) / 2$ no caso newtoniano, tornar-se-ia, no relativista, a diferencial de uma espécie de energia interna que iria sendo 'absorvida'. Nessa linha de raciocínio, a energia cinética continuaria sendo $m(v) v^{2} / 2$ e a energia interna total seria igual à diferença entre a energia $E(v), \mathrm{Eq}$. 9 , e a energia cinética $m(v) v^{2} / 2$, igual a $E(v)\left(1-v^{2} / 2 c^{2}\right)$. Não há, porém, indícios de que este racionalismo tenha apoio na realidade, para a qual $E-E_{0}$ parece ser a energia cinética da 
massa pontual em movimento, como usualmente admitido $[1,2]$.

\section{Comentários finais}

O que se procurou fazer aquí foi apresentar a Mecânica Relativista, num inespecificado sistema de coordenadas, sem nenhuma preocupação, como em [1,2], com a existência de outros sistemas. A consistência dos resultados endossa o ponto de vista de Sandin [4], admitido de forma geral na Eq. 5.

\section{Agradecimento}

O autor agradece ao colega Dr. René Armando Moreno a leitura de uma versão anterior do presente trabalho e os conselhos que a acompanharam.

\section{Referências}

[1] A.P. French, Special Relativity (W.W. Norton, 1968), caps. 1 e 6.

[2] H. Goldstein, Classical Mechanics (Addison-Wesley, 1951), cap.6.

[3] T. Theodorsen, Galilean Electrodynamics 6, 63 (1995).

[4] T.R. Sandin, Amer. J. Phys. 59, 1032 (1991).

[5] Nivaldo A. Lemos, Rev. Bras. Ens. Física. 23, 3 (2001). 